\title{
WINHAC - the Monte Carlo event generator for single $W$-boson production in hadronic collisions*
}

\author{
Wiesław Płaczek ${ }^{\dagger}$ \\ Marian Smoluchowski Institute of Physics, Jagiellonian University, \\ ul. Reymonta 4, 30-059 Kraków, Poland. \\ E-mail: Wieslaw.Placzek@uj.edu.pl
}

\begin{abstract}
We present the Monte Carlo event generator WINHAC for the charged-current Drell-Yan process, i.e. single $W$-boson production with leptonic decays in hadronic collisions. It features multiphoton radiation within the Yennie-Frautschi-Suura exclusive exponentiation scheme with $\mathscr{O}(\alpha)$ electroweak corrections. For the initial-state QCD/QED parton shower and hadronisation it is interfaced directly to PYTHIA. It implements the options for proton-proton, proton-antipronton and any nucleus-nucleus collisions. Moreover, it includes the polarized $W$-boson production and the neutral current Drell-Yan process, both at the Born level. Generation of weighted as well as unweighted (weight $=1$ ) events is possible. WINHAC has been thoroughly tested numerically and cross-checked with independent Monte Carlo programs, such as HORACE and SANC; in the so-called tuned comparisons, an agreement at the sub-per-mil level has been reached. It has also been used as a basic tool for developing and testing some new measurement methods of the Standard Model parameters at the LHC.
\end{abstract}

European Physical Society Europhysics Conference on High Energy Physics, EPS-HEP 2009,

July 16 - 222009

Krakow, Poland

${ }^{*}$ The work is partly supported by the program of the French-Polish cooperation between IN2P3 and COPIN No. 05-116 and by the EU Marie Curie Research Training Network grant under the contract No. MRTN-CT-2006-035505.

†Speaker. 


\section{Introduction}

Motivations for investigating the Drell-Yan processes, in particular that involving the $W$ bosons, at the Tevatron and LHC can be found e.g. in Refs. [1, 2, 3, 4, 5, 6, 7, 8, 9, 10].

In theoretical descriptions of the Drell-Yan processes reaching a sufficiently high precision for the Tevatron and LHC experiments requires including besides the QCD effects also the electroweak (EW) corrections, in particular the QED final-state radiation (FSR) [1]. It was shown in Ref. [11] that including $\mathscr{O}(\alpha)$ EW corrections may be not sufficient because the higher-order FSR effects can shift $M_{W}$ by $\sim 10 \mathrm{MeV}$.

Regions of a high transverse momentum of a charged lepton or a large $W$-boson transverse mass will be used at the LHC for various "new physics" searches. In such regions the $\mathscr{O}(\alpha) \mathrm{EW}$ corrections beyond the pure FSR can reach the size of 20-30\% [12,13]. Given high statistics of $W$ boson data expected at the LHC, these effects need to be included in order to have precise enough description of the Standard Model (SM) background.

Drell-Yan processes will also be treated at the LHC as the so-called 'standard candle' processes (detector calibration, normalisation, etc.). Therefore, their precise theoretical description is very important. In order to be fully exploited by the experiments, such a description ought to be provided in the form of a Monte Carlo event generator. Several semi-analytical programs as well as Monte Carlo integrators and event generators for Drell-Yan processes including QCD and/or EW effects have been developed in recent years, see e.g. Refs. [6, 10].

In this paper we briefly describe the Monte Carlo event generator WINHAC [14] dedicated to the charged-current Drell-Yan process. Section 2 is devoted to the QED FSR, while in Section 3 we describe inclusion of the $\mathscr{O}(\alpha)$ EW corrections. Finally, Section 4 contains summary and outlook.

\section{QED FSR}

Our first step in the development of WINHAC was to take into account effects of multiphoton FSR [15], based on the Yennie-Frautschi-Suura (YFS) exclusive exponentiation [16]. The total cross section for a parton-level process

$$
q_{1}\left(p_{1}\right)+\bar{q}_{2}\left(p_{2}\right) \longrightarrow W^{ \pm}(Q) \longrightarrow l\left(q_{l}\right)+v\left(q_{v}\right)+\gamma\left(k_{1}\right)+\ldots+\gamma\left(k_{n}\right),(n=0,1, \ldots)
$$

can be expressed as

$$
\sigma_{\mathrm{YFS}}^{t o t}=\sum_{n=0}^{\infty} \int \frac{d^{3} q_{l}}{q_{l}^{0}} \frac{d^{3} q_{v}}{q_{v}^{0}} \rho_{n}^{(1)}\left(p_{1}, p_{2}, q_{1}, q_{2}, k_{1}, \ldots, k_{n}\right),
$$

where

$$
\begin{aligned}
\rho_{n}^{(1)} & =e^{Y\left(Q, q_{l}, k_{s}\right)} \frac{1}{n !} \prod_{i=1}^{n} \frac{d^{3} k_{i}}{k_{i}^{0}} \tilde{S}\left(Q, q_{l}, k_{i}\right) \theta\left(k_{i}^{0}-k_{s}\right) \delta^{(4)}\left(p_{1}+p_{2}-q_{l}-q_{v}-\sum_{i=1}^{n} k_{i}\right) \\
& \times\left[\bar{\beta}_{0}^{(1)}\left(p_{1}, p_{2}, q_{l}, q_{v}\right)+\sum_{i=1}^{n} \frac{\bar{\beta}_{1}^{(1)}\left(p_{1}, p_{2}, q_{l}, q_{v}, k_{i}\right)}{\tilde{S}\left(Q, q_{l}, k_{i}\right)}\right] .
\end{aligned}
$$

The exponent in the above equation is the so-called YFS form factor in which all the infrared (IR) singularities coming from virtual and real photons are re-summed (in a gauge-invariant way) to 
the infinite order and properly cancelled. The rest of the equation is the accordingly reorganized perturbative series, which in the current version of WINHAC is truncated at $\mathscr{O}(\alpha)$. In particular, the $\bar{\beta}_{0}^{(1)}$ and $\bar{\beta}_{1}^{(1)}$ functions are the so-called non-IR YFS residuals corresponding to zero and one real hard-photon emission, respectively. This means that the former contains pure virtual corrections while the latter the pure real-photon ones. All details are given in Ref. [15].

Based on the above formula an appropriate Monte Carlo (MC) algorithm was constructed. We employed the importance sampling method by simplifying step-by-step the starting formula (2.2) until the simple enough probability density was obtained that could be generated with the use of basic MC methods. All these simplifications were compensated with corresponding MC weights. From these MC weights the full event weight was constructed. It can be used directly in weightedevent mode, e.g. for histogramming, or it can be applied in a rejection loop to construct weight $=1$ events in unweighted-event mode. In the weighted-event mode the program calculates for each event a large array of the MC weights corresponding to various effects, corrections, etc. The parton level cross section is convoluted with a product of two parton distribution functions (PDFs) corresponding to two colliding beams: proton-proton, proton-antiproton or any nucleus-nucleus. Values of Bjorken- $x$ 's of a quark and an antiquark participating in the hard process are generated from a 2-dimensional distribution, given by the product of two PDFs convoluted with a simplified ("crude") parton-level cross section, with the help of the adaptive cellular MC sampler FOAM [17]. Then, the quark and antiquark flavours are randomly chosen according to their relative contributions to the cross section.

After constructing the Monte Carlo event generator WINHAC we performed several numerical tests, both internal and external. The important external test was the comprehensive comparison with the independent MC program HORACE [11] which includes higher-order FSR corrections through a QED parton shower algorithm. We found the agreement between the two programs at the per-mil level, see Ref. [18] for details. This is very important since the two programs are based on very different approaches to the QED corrections. It suggests that major parts of the higher-order FSR corrections are included in both programs.

\section{EW corrections}

Strictly speaking, for the charged-current electroweak processes it is not possible to split the EW corrections into the pure QED and pure weak ones in a gauge-invariant way. However, one can find gauge-invariant subsets of QED corrections and separate them from the rest. Such a separation is in general not unique, as one can identify different subsets of gauge invariant QED corrections. In our definition of the QED FSR corrections described the previous section we used the prescription of Marciano and Sirlin (M\&S) given in Ref. [19]. Then we included the rest of the electroweak corrections for the FSR in the pole approximation (PA). These corrections have been provided to us in the form of the Fortran-code module by the SANC group [20]. Numerically they are small - at the level of $0.2-0.3 \%$ - and almost constant, so that they are not important for the $W$-mass measurements.

The main problem that appears in including the full $\mathscr{O}(\alpha)$ EW corrections (beyond PA) to the charged-current Drell-Yan process concerns the QED initial-state radiation (ISR), i.e. photon radiation from incoming quarks. In EW calculations the collinear ISR singularities are usually 
regularized with finite quark masses $m_{q}$. As a results, terms with $\ln \left(\hat{s} / m_{q}^{2}\right)$ appear in theoretical predictions. However, $m_{q}$ in this context is not a well-defined quantity, mainly because of the QCD effects. In fact, these singularities can be absorbed into the PDFs in a similar way as the ones in QCD, which is usually done in the $\overline{\mathrm{MS}}$ or DIS scheme, see e.g. Refs. $[12,13,20]$. QCD parton-shower generators, such as PYTHIA or HERWIG, usually generate also the QED ISR, however they do it in neither of the above schemes. So, combining such $\overline{\mathrm{MS}}$ or DIS-subtracted EW corrections with parton shower generators would result in some mismatch. Besides, the above schemes are not well suited for fully exclusive Monte Carlo generators, as they are defined for partly integrated quantities.

Therefore, we take a different approach to the QED ISR. We identify the QED gauge-invariant subsets of the ISR corrections and subtract them from the total $\mathscr{O}(\alpha)$ EW correction, leaving responsibility for their computation to parton-shower generators. In our opinion, it is better if the parton-shower generator treats both the QCD and QED ISR since the QED radiation is strongly affected by the QCD one. Of course, the parton-shower programs usually include the QED ISR in some approximation. However, the QED ISR corrections are small and flat for the main $W$-boson observables [1]. Moreover, at the LHC the $W$-boson distributions will have to be normalized to the corresponding $Z$-boson ones in order to avoid some large uncertainties, e.g. coming from the PDFs parametrisations, $W$-boson $p_{T}$-spectrum predictions, lepton energy scale determination, etc. In such ratios most of the QED ISR uncertainties will also cancel.

In WINHAC we have implemented three schemes for ISR subtraction: the one similar to the M\&S prescription for the FSR, the YFS-motivated scheme and the one from Ref. [21]. In fact, in the latter two schemes the ISR virtual plus soft real-photon corrections consist of the YFS form factor plus the terms $\sim(1 / 2) Q_{i}^{2}\left[\ln \left(s / m_{i}^{2}\right)-1\right]$, however our own formulae differ from those of Ref. [21] by some constant terms. Similar schemes can also be applied to the FSR and initial-final interferences - resulting in a separation of the $\mathscr{O}(\alpha)$ EW corrections into the 'QED-like' corrections and the 'weak-like' corrections. Such a splitting is, of course, not unique and it differs in various schemes by some constant terms. Our numerical tests show that the YFS-motivated scheme for the ISR is very close to the $\overline{\mathrm{MS}}$ scheme - the relative difference is $<0.05 \%$.

Similarly as in the case of the FSR, for the full $\mathscr{O}(\alpha)$ EW virtual corrections we use the Fortran-code modules from the SANC library [20]. Both virtual and real-photon corrections are implemented on top of the formula (2.2) through appropriate MC weights. In order to test their implementation, we performed numerical comparisons at $\mathscr{O}(\alpha)$ of WINHAC and the SANC MC integrator based on VEGAS. We have found a very good agreement - at the sub-per-mil level between two programs for the total cross sections and various distributions, see Ref. [22] for more details.

\section{Summary and outlook}

We have briefly presented the Monte Carlo event generator WINHAC for precision theoretical prediction of the charged-current Drell-Yan process, i.e. single $W$-boson production with leptonic decays in hadronic collisions. It includes multiphoton radiation in the YFS exclusive exponentiation scheme with the ISR-subtracted $\mathscr{O}(\alpha)$ EW corrections. For QCD/QED initial-state parton shower as well as hadronisation, it is directly interfaced with PYTHIA 6.4. It has been tested 
numerically and cross-checked to the high precision with independent programs, in particular HORACE and SANC. It has been used for developing new strategies of measuring the SM parameters $[5,7,8,9]$ and testing the mechanism of the electroweak symmetry breaking [3] at the LHC.

The current version, 1.30 , of WINHAC is written in FORTRAN7 7, however work is now in progress on re-writing it in $\mathrm{C}++$. A similar program, called ZINHAC [23], for the neutral-current Drell-Yan process is being completed now - in $\mathrm{C}++$.

\section{References}

[1] U. Baur, S. Keller, and D. Wackeroth, Phys. Rev. D59 (1999) 013002, hep-ph/9807417.

[2] S. Haywood et al., Electroweak physics, hep-ph/0003275.

[3] M. W. Krasny, S. Jadach and W. Płaczek, Eur. Phys. J. C44 (2005) 333, hep-ph/0503215.

[4] J. Alcaraz et al., A Combination of Preliminary Electroweak Measurements and Constraints on the Standard Model, 2006, hep-ex/0612034.

[5] M. W. Krasny, F. Fayette, W. Płaczek and A. Siódmok, Eur. Phys. J. C51 (2007) 607, hep-ph/0702251.

[6] TeV4LHC-Top and Electroweak Working Group, C. E. Gerber et al., 0705.3251 [hep-ph].

[7] F. Fayette, M. W. Krasny, W. Płaczek, and A. Siódmok, Eur. Phys. J. C63 (2009) 33, 0812.2571 [hep-ph].

[8] F. Fayette, Strategies for precision measurements of the charge asymmetry of the $\mathrm{W}$ boson mass at the LHC within the ATLAS experiment, 2009, 0906.4260 [hep-ex], Ph. D. thesis.

[9] A. Siódmok, M. W. Krasny, F. Fayette and W. Płaczek, W-boson mass measurement at the LHC, in preparation.

[10] C. Buttar et al., Standard Model Handles and Candles Working Group: Tools and Jets Summary Report, 2008, 0803.0678 [hep-ph].

[11] C. M. Carloni Calame, G. Montagna, O. Nicrosini and M. Treccani, Phys. Rev. D69 (2004) 037301.

[12] S. Dittmaier and M. Krämer, Phys. Rev. D65 (2002) 073007, hep-ph/0109062.

[13] U. Baur and D. Wackeroth, Phys. Rev. D70 (2004) 073015, hep-ph/0405191.

[14] W. Płaczek and S. Jadach, WINHAC version 1.30: http://cern.ch/placzek/winhac.

[15] W. Płaczek and S. Jadach, Eur. Phys. J. C29 (2003) 325, hep-ph/0302065.

[16] D. R. Yennie, S. Frautschi and H. Suura, Ann. Phys. (NY) 13 (1961) 379.

[17] S. Jadach, Comput. Phys. Commun. 152 (2003) 55, physics/0203033.

[18] C. M. Carloni Calame, S. Jadach, G. Montagna, O. Nicrosini and W. Płaczek, Acta Phys. Polon. B35 (2004) 1643, hep-ph/0402235.

[19] W. J. Marciano and A. Sirlin, Phys. Rev. D8 (1973) 3612.

[20] A. Arbuzov et al., Eur. Phys. J. C46 (2006) 407, hep-ph/0506110.

[21] D. Wackeroth and W. Hollik, Phys. Rev. D55 (1997) 6788.

[22] D. Bardin, S. Bondarenko, S. Jadach, L. Kalinovskaya and W. Płaczek, Acta Phys. Polon. B40 (2009) 75, 0806.3822 [hep-ph].

[23] A. Siódmok and W. Płaczek, ZINHAC: http://th-www.if.uj.edu.pl/ZINHAC/. 